\title{
Optical Vector Signal Analyzer Based on Differential Direct Detection
}

\author{
J. Li ${ }^{(1)}$, K. Worms ${ }^{(1)}$, P. Vorreau ${ }^{(1)}$, D. Hillerkuss ${ }^{(1)}$, A. Ludwig $^{(4)}$, R. Maestle ${ }^{(3)}$, \\ S. Schüle ${ }^{(2)}$, U. Hollenbach ${ }^{(2)}$, J. Mohr ${ }^{(2)}$, W. Freude ${ }^{(1)}$, J. Leuthold ${ }^{(1)}$ \\ (1) Institute of Photonics and Quantum Electronics, University of Karlsruhe, \\ and ${ }^{(2)}$ Institute for Microstructure Technology, Forschungszentrum Karlsruhe $\mathrm{GmbH}$, \\ Karlsruhe Institute of Technology (KIT), D-76131 Karlsruhe, Germany / Email: ing-shi.li@ipq.uka.de \\ (3) Agilent Technologies, Herrenbergerstr. 130, D-71034 Böblingen, Germany \\ (4) Now with German Aerospace Center (DLR), Oberpfaffenhofen, D-82234 Weßling, Germany
}

\begin{abstract}
A$ versatile optical vector signal analyzer based on a differential direct detection scheme is presented. The analyzer measures phase, intensity, eye diagrams and constellations. The performance is demonstrated for a 112 Gbit/s PoIMUX DQPSK signal and other formats.
\end{abstract}

\section{Introduction}

Various modulation formats $s^{1,2,3}$ are under discussion for $100 \mathrm{Gbit} / \mathrm{s}$ transmission and beyond, including RZ, DPSK, QPSK, QAM combined with polarizationmultiplexing (PoIMUX). The difficulty though is that no cheap and versatile vector signal analyzer is available that allows a full characterization of the constellation diagrams, the eye diagrams and the time dependency of optical phase and intensity. Practical tools based on coherent detection schemes or on optical sampling techniques have recently come to the market ${ }^{4,5}$. Yet, while all of these tools work well, they are expensive since they rely either on a narrow-bandwidth coherent laser that is limited in its tuning range, or on modelocked laser sources.

It has recently been shown that by means of differential direct detection schemes one can recover both phase and intensity of an optical signal ${ }^{6,7}$.

In this paper, we demonstrate an optical vector signal analyzer (OVSA) that analyzes PoIMUX signals by employing pairs of delay interferometers with delay $\tau$. We recover the phase difference and the intensity of optical signals as a function of multiples of $\tau$. The delay $\tau$ is tuned according to the bitrate and the requested number of samples per symbol (SpS). Experimentally we demonstrate the successful reception of a $112 \mathrm{Gbit} / \mathrm{s}$ (i.e., $28 \mathrm{GBd}$ ) PoIMUX DQPSK signal, and of other formats. The temporal resolution is limited by the fine adjustment of $\tau$ and by the bandwidth of the photodiodes.

\section{Operation Principle}

The schematic setup of an OVSA for analyzing PolMUX DQPSK signals is shown in Fig. 1(a). The input signal is split into two orthogonally polarized beams with a polarization beam-splitter (PBS), and processed by pairs of tunable optical delay interferometers (TODI) having (orthogonal) cosine and sine characteristics, respectively. The TODIs are connected to balanced receivers. The complex optical input signal with an envelope $r(t)=|r(t)| \exp [\mathrm{j} \phi(t)]$ is split to two paths, and sent to two TODIs. In the TODI, the signal is further split to two arms having a delay difference $\tau$. Delayed and non-delayed signals

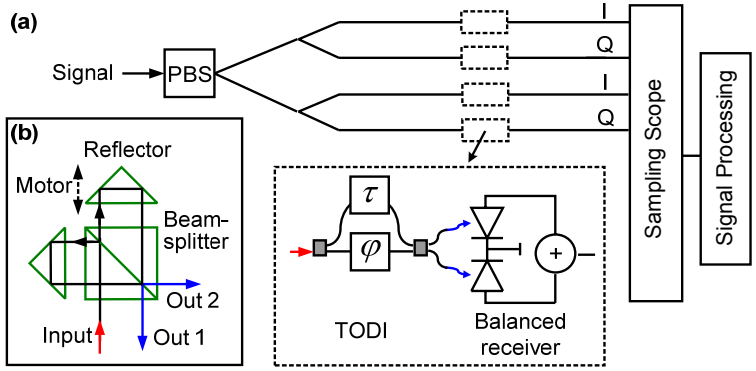

Fig. 1: (a) Optical vector analyzer scheme. (b) Freespace optics layout of tunable optical delay interferometer (TODI)

interfere at the output ports. Orthogonal interferometer characteristics are achieved by a relative phase offset in the arms of one TODI with respect to the other TODI amounting to $\varphi=\pi / 4$ and $\varphi=-\pi / 4$, respectively. The balanced receivers' output currents of the in-phase (I) and quadrature (Q) TODI are

$$
\begin{aligned}
& i_{I}=k|r(t)||r(t-\tau)| \cos \left(\Delta \phi(t)+\frac{\pi}{4}\right), \\
& i_{Q}=k|r(t)||r(t-\tau)| \sin \left(\Delta \phi(t)+\frac{\pi}{4}\right),
\end{aligned}
$$

where $k$ is a constant, and $\Delta \phi(t)=\phi(t)-\phi(t-\tau)$. These output currents then are electrically sampled with an Agilent sampling oscilloscope. An off-line software tool then extracts the complex current ${ }^{7}$

$$
i=i_{I}+\mathrm{j} i_{Q} \text {. }
$$

An approximation for the amplitude $|r(t)|$ and an exact relation for the phase $\phi(t)$ at time $t=t_{0}+n \tau$ (with respect to an arbitrary reference phase at $t_{0}$ ) leads to ${ }^{7}$

$$
\begin{aligned}
r\left(t_{0}+n \tau\right)= & \left|i\left(t_{0}+n \tau\right) \cdot i\left(t_{0}+n \tau+\tau\right)\right|^{\frac{1}{4}} \\
& \times \exp \left(\mathrm{j} \sum_{m=1}^{n}\left(\arg \left(i\left(t_{0}+m \tau\right)\right)-\frac{\pi}{4}\right)\right) \exp \left(\mathrm{j} \phi\left(t_{0}\right)\right) .
\end{aligned}
$$

\section{Experimental Setup}

Our TODI is a free space interferometer. As shown in Fig. 1(b), one of the paired reflectors is mounted on a piezo-actuator which provides $2 \times 2 \mathrm{~nm}$ increments for phase and time delay adjustments. The tunable delay range is $\tau=0 \ldots 100 \mathrm{ps}$. The beamsplitter is carefully chosen so that it performs similarly for both orthogonal polarization states. The setup can be miniaturized with our LIGA micro-optical bench technology ${ }^{8}$. 

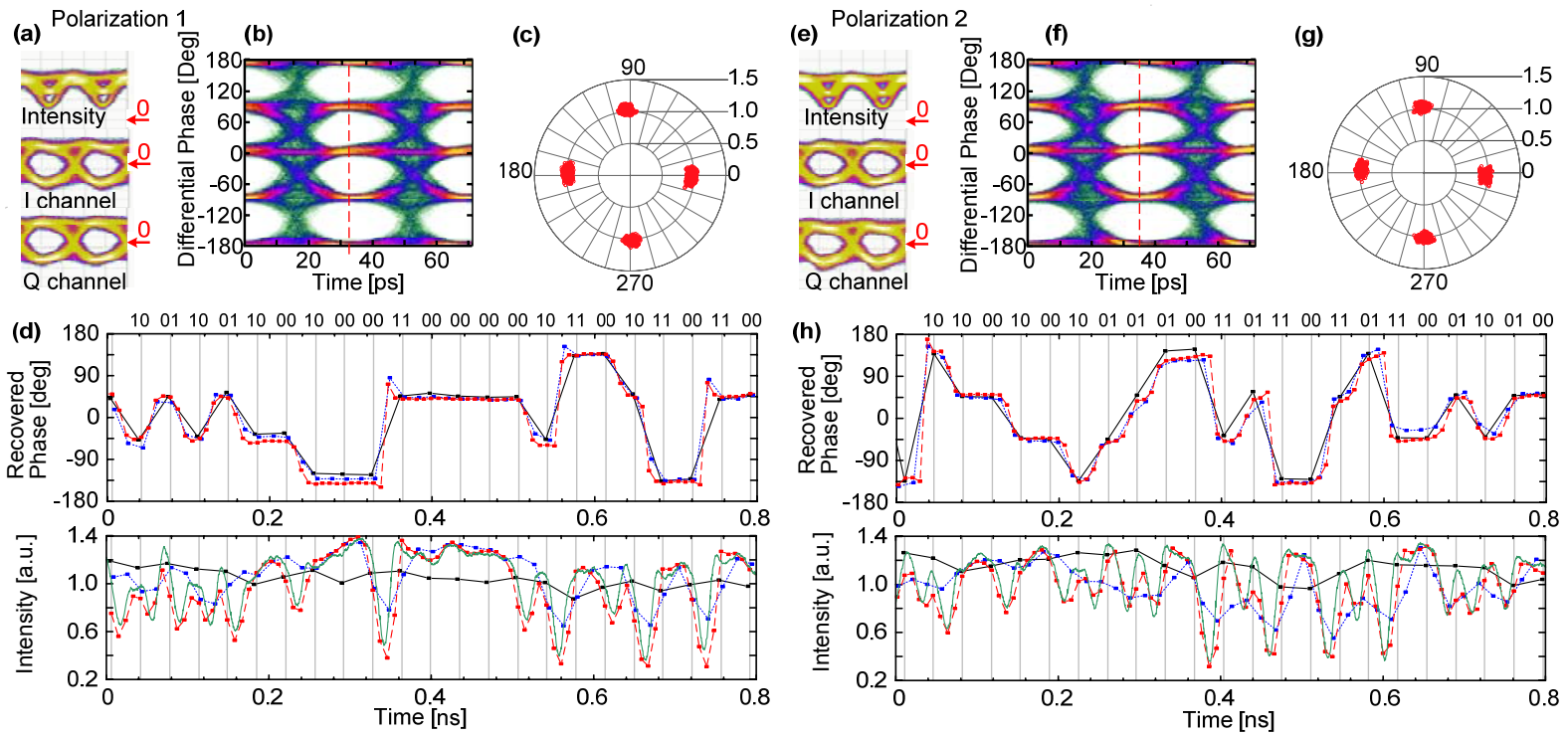

Fig. 2: Detection of 2,085 symbols. (a) \& (e): Measured intensity and $I \& Q$ channel eye diagrams; (b) \& (f): differential phase eye diagrams with sampled once per symbol $(\mathrm{SpS}=1)$; (c) \& $(\mathrm{g})$ : constellation diagrams of DQPSK signal for each of two orthogonal polarizations; (d) \& (h): recovered intensity and phase as a function of time with $\mathrm{SpS}=1(-\cdot-)$, $\mathrm{SpS}=2$ $(\ldots-\ldots)$ and 4 (--- ---); Directly measured intensity $(-)$ shown for comparison. Symbol frame centers marked in grey $(\mid$ )

\section{Experimental Results}

An optical $112 \mathrm{Gbit} / \mathrm{s}$ PoIMUX DQPSK signal with a symbol rate of $28 \mathrm{GBd}$ at $\lambda=1,555.75 \mathrm{~nm}$ and a pseudo-random sequence length of $2^{11}-1$ is launched into the OVSA. The polarization in front of the PBS was matched to the vertical and horizontal polarization of the receiver pairs, respectively. We first set the delay to 35.7 ps corresponding to the symbol duration of a $28 \mathrm{GBd}$ signal. The I and $\mathrm{Q}$ waveforms are then detected with photodiodes (cut-off frequency about $75 \mathrm{GHz}$ ) and sampled by an Agilent sampling oscilloscope, Fig. 2(a)(e). The waveforms are subsequently off-line processed. The differential phase, the recovered phase and the intensity can be calculated with equations (2) and (3). By overlaying the recorded 2,085 symbols, the differential-phase eye diagrams can be plotted, Fig. 2(b)(f) for the two polarizations,
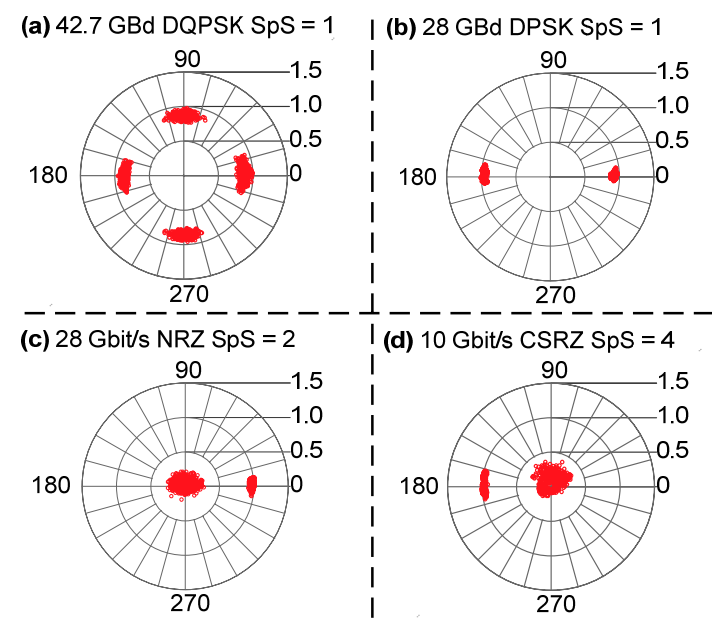

Fig. 3: Constellations of (a) $42.7 \mathrm{GBd}$ DQPSK $\mathrm{SpS}=1$, (b) $28 \mathrm{GBd}$ DPSK SpS $=1$, (c) $28 \mathrm{Gbit} / \mathrm{s}$ NRZ SpS $=2$, and (d) $10 \mathrm{Gbit} / \mathrm{s}$ CSRZ SpS $=4$. respectively. The constellation diagrams of the PolMUX DQPSK are plotted in Fig. 2(c)(g). The fully recovered phase and amplitude of the signal are plotted in Fig. 2(d)(h). The result of the sampling process with $\mathrm{SpS}=1$, i.e., a delay of $\tau=35.7 \mathrm{ps}$ is shown as $(--)$. Setting the delay to 17.85 ps and 8.9 ps, i.e., to SpS $=2(\ldots \cdots)$ and $\mathrm{SpS}=4(---\cdot--)$, provides more accurate results. The recovered phase at $\mathrm{SpS}=4$ reveals the most details, and the recovered intensity at $\mathrm{SpS}=4$ matches best with the measured intensity $(\longrightarrow$ ) that has been recorded as a check.

We tested the OVSA with other modulation formats at various bitrates, namely with a DQPSK signal at $42.7 \mathrm{GBd}$, a DPSK signal at $28 \mathrm{GBd}$, an NRZ signal at $28 \mathrm{Gbit} / \mathrm{s}$, and a CSRZ signal at $10 \mathrm{Gbit} / \mathrm{s}$, see Fig. 3. The constellation diagrams are plotted with the samples positioned at the symbol center.

\section{Conclusions}

The design of a format-transparent optical vector signal analyzer having a tunable sampling rate and bitrate is explained. Its function is demonstrated for 112 Gbits/s PolMUX QDPSK signals and other formats.

\section{Acknowledgement}

This work is supported by the Agilent University Relation Program and Karlsruhe School of Optics and Photonics (KSOP).

\section{References}

1 P.M. Watts et al., IEEE PTL 17, pp. 2206, Oct. 2005

2 M. Yoshida et al., Opt. Expr. 16, pp. 829, Jan. 2008

3 A. Ellis, OFC 2009, paper 0MM4, March 2009

4 Agilent N4391A Optical Modulation Analyzer, March 2009

5 H. Sunnerud et al., OFC 2009, paper OThF4, March 2009

6 N. Kikuchi et al., ECOC 2006, paper Th444, Sept. 2006

7 X. Liu et al., Opt. Expr. 16, pp. 792, Jan. 2008

8 S. Schüle et al., Photonics Europe, SPIE Proc., 6992-1. April 2008 